\title{
Thinking About the Physical Activities for Preschool Children and System Reconfiguration of the Movement Programs
}

\author{
Zhuang Bi ${ }^{1}$, Ren $\mathrm{Qi}^{2}$, Li Mengning ${ }^{3}$, Jing Pengfei ${ }^{4}$, Zhang $\mathrm{Yi}^{5}$ \\ ${ }^{1}$ Department of Public Sports, Guangzhou College of Commerce, Guangzhou, China \\ ${ }^{2}$ Department of Sports Science, South China Normal University, Guangzhou, China \\ ${ }^{3}$ Department of Physical Education, ChangAn Experimental Middle School of Dongguan, Dongguan, China \\ ${ }^{4}$ Department of Physical Education, Taishi Senior High School of Taishan, Jiangmen, China \\ ${ }^{5}$ Department of Graduate School, Guangzhou Sport University, Guangzhou, China
}

Email address:

zhuang9851@163.com (Zhuang Bi)

\section{To cite this article:}

Zhuang Bi, Ren Qi, Li Mengning, Jing Pengfei, Zhang Yi. Thinking About the Physical Activities for Preschool Children and System Reconfiguration of the Movement Programs. International Journal of Sports Science and Physical Education. Vol. 4, No. 1, 2019 , pp. 9-17. doi: $10.11648 /$ j.ijsspe.20190401.12

Received: January 24, 2019; Accepted: April 12, 2019; Published: May 17, 2019

\begin{abstract}
Preschool activities for children is a period of human life skills from the beginning, its main form of expression are physical activities. It is important and useful to cultivate children's physical activities awareness, skills and habits. It is of great significance to improve children of body and psychology development; cultivate physical activities habits;increase level of physical fitness. According to special require of Ministry of Education "3-6 years old children study and development guide"in our country, we make analysis and thinking for children physical activities which have problems about development present situation and set up to kindergarten physical activities contents system reconstruction:1) Stratification and progressive construction of children's sports activity content system; 2) Screening and establishing a measurement index system for children's sports activities; 3) Creating a suitable sports game environment; 4) Establishing a teaching organization system for children's sports activities; 5) Creating a suitable sports game environment Establishing a teaching organization system for children's sports activities; 6) Cultivation of children's behavioral norms and rules awareness; 7) Integration of sensory integration training in early childhood sports activities. To provide reference for the reform and development of sports activities in preschool education.
\end{abstract}

Keywords: Preschool Children's Physical Activities, System, Reconfiguration

\section{Introduction}

Many countries regard preschool education as the foundation of their entire education systems. Since the 1980s, strengthening preschool education has become one of the main goals for future

education in the world. Preschool education has gradually been included in the compulsory education and lifelong education system. As early as 1996, the Nursery Work Regulations" promulgated by the National Education Commission clearly pointed out that the main task of kindergartens is to "implement the principle of combining childcare and education, and to educate children on the comprehensive physical, intellectual, moral and aesthetic development which is reflected in the Ministry of Education's promotion of harmonious development of mind and body. Guidelines for Kindergarten Education [1]. The National Education Commission puts "body" in first place, as the level of sports development in early childhood directly lays the foundation for the level of sports development in adulthood. It can be seen that physical education in the early childhood stage is the first and the key point. Because the early childhood stage is the foundation period of physical and psychological development, there is no moral, intellectual or aesthetic development without it. 


\section{Function and Definition}

\subsection{The Role of Children's Sports Activities in Physical and Mental Development and Quality Education}

\subsubsection{Characteristics of Children's Physical and Mental Development}

The laws governing the development of children's bodies are sequential, unbalanced, staged, and wholly individualised. However, due to differences in individual genetics and living environment, each child's physical and mental development may have its own uniqueness in terms of expression, content and level. Taking the development of young children's movements as an example, the development of large bones and large muscles is first developed, and then the development and coordination of small bones and small muscle groups. Therefore, in the coordinated development of bones and muscles, young children experience the changes in various stages. The process of exercise development manifests itself as a constant sequence. That is to say, the development of young children's movements is based on topdown development. They can first raise their heads, raise their chests, turn over, and then sit, and then they can complete large muscle movements such as standing erect and independent walking. The large muscle movements close to the torso develop first. Small muscle activity at the distal end of the limbs follow, and then there may be fine movements of small muscles leading to actions such as writing, painting, and hand picking. The coordination ability of the eye and hand is gradually improved [2]. The speed of physical and mental development of children at different ages is not completely consistent with time. Therefore, the content of sports activities should be consistent with the characteristics of physical and mental development of children of all ages. From the perspective of psychological development, young children can perceive the world and explore the external environment through various sports activities. Accumulating experience through exercise can lay a foundation for their psychological development and social adaptability.

\subsubsection{The Role of Sports Activities in Quality Education for Children}

The three reforms of kindergarten education in modern China have gone through four major stages: the 1952 "Interim Teaching Outline for Kindergartens", the "Programme for Kindergarten Education" in 1981, and the "Guidelines for Kindergarten Education (Trial)" in 2001 and 2012. The "Guidelines for the Learning and Development of Children Aged 3-6" (hereinafter referred to as the "Development Guide") is the guideline [3]. Their overall goal is the same; that is, to promote the overall physical, intellectual, moral and aesthetic development in children, and the physical always ranks first. From the perspective of children's physical and mental development, preschool children are at the beginning of life, the functions of various organs and systems of the body have not yet fully developed, and the pre-school period is a period of rapid growth and vigorous development, which promotes the healthy development of children. It is the primary task of this period. The National Medium- and Long-Term Education Reform and Development Plan (2010-2020) emphasizes that "preschool education is important for early childhood habits, intellectual development, and physical and mental health [4]. Follow the rules of children's physical and mental development, adhere to the scientific method of education, and ensure the healthy growth of young children." Sporting activities have multiple educational functions, which do not only aid physical development of young children; but can also promote their formation of good character, communication skills and promote social development. Aristotle pointed out in his seventh volume of Political Science that "sports should be the first education" [5]. Chen Heqin, a famous preschool educator in China, said: "Children are born to be active and play games." From the related research and writings on children's games (including sports games), it can be seen that children's sports in early childhood quality education play a very important role.

\subsection{The Connotation of Children's Sports Activities}

\subsubsection{Understanding of Children's Sports Activities in Major Countries of the World}

Children's sports are essentially the process of raising and educating the children's body, not the sports we generally understand. Therefore, in many pre-school education programs or curriculum guides, many children's sports activities are included in the health category, and the physical nurture of the children is completed via games or sports activities.

In the 20th century, the United States paid attention to the overall development of young children, including social development, cognitive development, emotional development and physical development. In April 1993, the US government promulgated the "2000 Target: American Education Act," stating that "children will receive the nutrition, physical exercise, and health care required for physical and mental health when they enter school". The 2007 Early Improvement Act states that it provides a learning environment for children from low-income families to support their development in language, literacy, mathematics, science, social and emotional understanding, creativity, physical motor skills and learning methodology.

In 2007, the UK Ministry of Education and Skills introduced the Early Stages of Early Childhood, establishing a unified game-based framework for early childhood learning and development. The value of preschool education in the development of children's sports is to promote the comprehensive development of children's emotional, physical, social and intellectual aspects, and to help children prepare for school.

In the revision of the "Kindergarten Conservation Outline" conducted by Japan in 1999, the field of kindergarten education was revised into distinct categories: health, interpersonal relationships, nature, language, painting and crafts, and musical rhythm. In the field of health, the emphasis is on the role of games, and targets child health 
education activities through nature and the outdoors.

In 2003, the National Children' s Charter of India put forward several requirements for pre-school education: children' s survival, life and freedom; promoting high standards of health and nutrition; ensuring minimum needs and life safety; games and entertainment [6].

The purpose of the sports activities of the French maternal school is to enable children to practice their motor skills by providing them with the exercise conditions needed to maintain their health, and to promote the development of children's adaptability and cooperation. The activities of the sports activities include: full-body motor function activities such as walking, running, jumping, crawling, climbing, throwing, carrying, maintaining balance; sports coordination activities such as agile games, speed games, confrontation games, dance and round dance with singing; human body performance activities via simple performances across all actions.

The basic methodology for preschool education in Russia is to promote the development of children's physical, intellectual, moral and artistic abilities through games and activities. Russian preschool institutions recognize that physical activity is different from sports games and that they must institutionalize sports in early childhood. Therefore, Russian kindergartens are generally large in size and rich in sports equipment. In order to satisfy the child's activity nature, the kindergarten usually arranges sports activities to combine traditional sports with non-traditional. Common sports training programs include morning exercises, aerobics, racquet games, table tennis, sledding, running stairs, skipping and running. These different kinds of sports are arranged for children across different ages. Russian kindergartens attach great importance to running training and also attach great importance to endurance training. Attaching importance to the combination of sports and games is also one of the characteristics of their preschool education [7].

China has always put the physical at the forefront of children's sports activities. In the general rules of the "Kindergarten Work Regulations", the "Education for the comprehensive development of children's physical, intellectual, moral, and aesthetic aspects" is proposed in the General Regulations. The goal is to "promote the development and function of children's normal development and function, and enhance physical fitness." The Guidelines for Kindergarten Education (Trial) and the Guide for Learning and Development for Children aged 3-6 in 2012 began to put children's sports activities under the health field, but none of them gave much explanation about children's sports.

\subsubsection{Definition of Children's Sports Activities}

For a long time, there have been few theoretical studies on children's sports activities in China, and the concept of children's sports activities is chaotic. At present, the experts who have more influence in preschool education in China have different understandings of children's sports, and there are "children's physical education", "Children's sports", "children's physical activity" and "children's body movements", among other expressions. The concept and scope of the definition are unclear.

Preschool education expert Xu Zhuoya called children's sports "kindergarten sports." The basic content of kindergarten sports includes basic movements, basic gymnastics and sports games. The rhythmic, dancing and expressive and creative physical activities are also incorporated into the basic content of kindergarten sports [8].

Liu Xin, a preschool education expert, puts "sports activities and physical exercise (physical exercise)" in parallel, which means that the two have different connotations. The belief here is that sports activities are an integral part of social culture, and children' s sports are different from school sports, thus creating a special field of education that integrates child care and education. Physical exercise has two functions or values for the development of preschool children: promoting the coordination development of children's bodily functions and motor functions; and using physical movement as a means to promote the healthy development of children's psychology. [9] Liu Xin called infant sports "physical exercise", mainly by exerting certain stimulation on the body of young children (That is, the stimulation of exercise). A certain amount of exercise stimulation acts on the body of the child, so that the body of the child is subjected to the corresponding physiological load. The regularization of the stimulation causes the child's internal body to constantly adjust and gradually produce adaptive changes, so that the body is in shape, the structure and function have been improved and improved.

Pang Jianping and Liu Qian put forward that "preschool children's sports is a special field of education that integrates conservation and education on the basis of the characteristics and laws of children who are 0-6 years old." [10].

Wang Zhanchun and Chen Yuqi believe that: "Children's sports follow the rules of the body's growth and development to enhance physical fitness and improve health. It is a series of educational activities aimed at promoting the comprehensive and harmonious development of children's mind and body." [11].

Comparing the various expressions of children's sports, this paper believes that they have not clarified the goal of children's physical development in essence. Therefore, this paper proposes that kindergarten sports should be called "children's sports activities".

"Children's sports activities", this formulation not only helps to distinguish between physical education in the primary and secondary schools, but also realizes the concept of "young convergence" for the following reasons:

1) Physical education should include elements such as physical exercise, games and Educational guidance. The expression of "children's sports" is too focused on sports and educational guidance. It is inevitable to strengthen the awareness of training, with competitive meaning, and to dilute the joy of the game. The concept of "children's physical activity" emphasizes physical exercise and physical exercise. The exercise effect is related to the growth and 
development of the child's body and physical fitness, and a focus on sports education is unnecessary.

2) Early childhood sports include physical education, sports and competitions. The development of "children's sports" in kindergartens tends to lead to the introduction of the content, methods and skills of competitive sports directly into the physical activities of kindergartens, which leads to the phenomenon of destructive encouragement.

3) The term "children's sports activities" has the meaning of physical exercise, and of play

and happiness. In the early childhood stage of sports, in addition to the goal of enhancing physical fitness, it should be biased towards stimulating and maintaining the interest of young children to participate in physical exercise through happy activities. At the same time, happy sports activities can satisfy the emotional needs of young children, thus promoting their psychological and physical development.

4) "Children's sports activities" includes the experiential process of activities and games. In sports activities, according to the characteristics of the children themselves, the activity plot is infiltrated, adapting to the psychological needs of the children to imitate and participate in the adult activities and the socialization needs of the children, so as to trigger the children to participate in the exercise through exploratory activities (such as rotating to explore the movement of different parts of the body; using methods of throwing and picking to explore the common movements of the body and the object; rolling over to experience the relationship between the body and the space, etc.), without focusing on the skill learning. Motor skills learning is the goal of physical education at the school stage.

In summary, this paper believes that children's sports activities are the process of cultivating and developing the basic physical activity ability of young children (3-6 years old).

\section{Results and Discussion}

\subsection{The Goal of Children's Sports Activities is Unclear and Lacking in Specific Content}

From the perspective of children's physical and mental development, preschool children are at the beginning of their life, the functions of various organ systems of the body have not yet fully developed, and the pre-school period is a period of rapid and vigorous growth and development, promoting the healthy development of children; It has become the primary task of this period. The "physical" referred to in preschool education is the comprehensive and coordinated development of the body. In early childhood, "physical" should not be understood as the undertaking of sports in daily life, but should be understood as the process of developing the body of the child, a process that cannot be similarly carried out in adults. Therefore the sports required for such development should be based on the cultivation of basic human activities. The content of children's sports activities should be consistent with the goal of laying a good quality foundation for children's follow-up learning and lifelong development, in order to promote children's physical, intellectual, moral and aesthetic aspects. The task of comprehensive and coordinated development as the core [12]. From the current situation of children's sports activities, the objectives of the activities are not clearly unified, the requirements are not specific; the content of the activities lacks certain continuity, system and flexibility, and there is a certain lack of direction. Because there is no unified standard content and syllabus for kindergarten sports activities in China, there are many serious deviations in the practice of kindergarten physical education, which makes the goals of children's sports activities easy to be associated with general sports, leading to the activities of adult physical education in kindergartens, while ignoring the cultivation, upbringing and cultivation of children's basic activities.

In response to these shortcomings and problems, the Ministry of Education's "Development Guide" was formulated in 2012, based on the basic laws and characteristics of learning and development of 3-6 years old children. It puts the requirements and goals of physical development of children in three groups, that is, the ability to develop child balance, strength and endurance, sensitivity and coordination in physical health. However, the "Development Guide" only proposes the target requirements, and lacks specific guidelines for the content of sports activities for children aged 3-6 years, especially for the characteristics of different age groups. The best course of action to choose for development needs to be refined. A group of 3-4 year old children were used to conduct a 6month experimental study, the results of which show that the sports activities designed according to the orientation concept will improve the quality of the 3-4 year old children's movements, stimulate their sports potential beyond that of the current early childhood sports. The current activity content neglects a specialised focus on children's sports experience, the content is singlular, and lacking in a diverse range of skills and neither the load intensity of practice action nor the construction of mental environment are emphasized. [13].

The disorder of the goals of children's sports activities and the disconnection of content puts the arrangement of kindergarten sports activities in a chaotic state.

\subsection{The Content and Form of Children's Sports Activities are Relatively Random}

At present, the sports activities based on the development of children's basic activities are the basis of their future learning. Based on comprehensive analysis of the current status of sports activity system and the construction of scientific children's sports activities, the overall organization of children's sports activities, the scientific monitoring of the number of activities, their selection, cultivation, and their subsequent research on the measurement and evaluation are extremely weak and insufficient compared to sports research in primary and middle schools. In many kindergartens, the content and form of physical education classes lack scientific 
basis or randomness. The content of sports activities cannot be arranged according to the characteristics of children's physical and mental development, as is the trend in adult education.

There is a consensus in the field of international children's education: premature technical training can hinder children's imagination and creativity. Many teachers lack comprehensiveness in the understanding of the "physical". Under the influence of traditional sports, they all recognize the sports of kindergartens from the level of competitive sports, and lack cultivation and upbringing of basic activities for young children. Seefeldt believes that "unless the individual's multiple motor skills are fundamentally developed, their level of motor skills will be difficult to develop to the advanced level of the 'pyramid' - the level of proficiency required to achieve athletic, competitive and dance motor skills." He further suggested that "children should develop a broad skill base at the early to mid-stage of childhood before it is possible to develop into higher-level motor skills at the 'pyramid'“'[14]. Many athletic sports projects have entered kindergartens, such as basketball teaching, football teaching, and even some golf teaching has appeared in kindergartens. Their fine muscle development is still at a relatively low level. It is too early to carry out technical training, so that children who have not yet developed their muscles find only unsatisfactory results. It is like letting a thin child take a heavy burden and letting him compete with himself. It is easy to make children feel frustrated 、 affecting self-confidence and interest. This situation is caused by the lack of guidance on the proper content of children's sports activities, and the choice of teaching content is highly random.

\subsection{Testing and Evaluation of Children's Sports Activities Adulthood}

Accuracy and effectiveness of testing and evaluation criteria for children's physical activity play a decisive role in the analysis and evaluation of children's physical development. Since 2000, China has conducted a physical health survey for Chinese citizens aged 3 to 69 every five years. The National Physical Fitness Measurement Standard (Children's Part), developed by the State Sports General Administration, provides a basis for the analysis and evaluation of children's physical development. Test indicators include body shape and quality indicators including height and weight; quality tests include 10 meter relay running, standing long jump, tennis ball throwing, continuous jumping, flexibility while sitting and balance beam walking [15]. At present, the "National Physical Fitness Measurement Standards (Children's Part)" adopts a unified test program and gives corresponding evaluation criteria for children of different ages. From the current literature, a large number of documents involving the testing and evaluation of children's sports activities use these test items as the basis for evaluation.

The requirements and goals of the child's physical development proposed by the Ministry of Education's
"Development Guide", that being the development of "children's balance, strength and endurance, sensitivity and coordination, etc.", should be fully tested and evaluated by machine, simply using quantitative evaluation is not enough. In the National Fitness Measurement Standards (Children's Part), much of the children's physical activity testing and evaluation programs are adult in approach and tendency, and obviously fail to meet the age-division learning and child movement development requirements of the Development Guide. Therefore, it is necessary to develop new measurement and evaluation criteria according to the characteristics of different age groups within young children. In addition, according to the development requirements of different physical qualities, test items can be diversified, and the activity ability items and evaluation criteria such as balance, strength and endurance, sensitivity and coordination ought to be determined according to the age characteristics of the children, forming a comprehensive assessment of these qualities. Additionally, the evaluation index of endurance quality and its judgment standard system. For example, there are many test items for assessing balance quality, such as standing with one eye open on one foot, standing on one foot, walking on a balance beam, and children of certain ages can choose suitable test items such as closing eyes on one foot reflecting the balance ability of 3-4 years old children. Tests to determine the development of children in this age group influence the evaluation criteria for children of all ages. In addition, some test items can improve test scores through repeated project exercises, such as walking the balance beam, so that the test results may not truly reflect the actual level of the child's quality. Standing with some combination of eyes closed on one foot only relies on the coordinated movement of the balance receptors of the vestibular organs of the brain and the muscles of the whole body. The duration the child maintains the center of gravity on the one-legged support surface can reflect the strength of the balance ability. Therefore, the balance action test as a closed eye and one foot stand can be used instead of walking the balance beam, as it can more effectively reflect the balance quality of the child realistically and objectively.

\subsection{Insufficient Health Care and Safety Measures for Children's Sports Activities}

Not all sports can promote the healthy growth of young children, therefore ensuring that children's health care and safety in sports activities is a priority for the effectiveness of sports activities. In the activities of children's sports, educators should pay attention to the scientific arrangement and monitoring of the amount of exercise according to the characteristics of children's physical and mental development, prevent all kinds of accidental injuries in children during exercise, and carry out safety education and protection for young children. Educators should gradually develop young children's self-protection awareness and behavior in sports activities and avoid and reduce unsafe behaviors. The amount of exertion that the body bears when the child is active depends on the time, intensity, and density 
of the activity. Children's sports activities should maintain a suitable level of exertion. If the exertion is too small, the expected targets of the physical activity will not be achieved. If the amount of exertion is too large, it will increase the physical load on the child and even cause injury. At present, the guidelines for activities in this area are not clear enough, lack operational norms, and mainly rely on the observation of educators. Combined with the current development of sports science, it can provide appropriate guidance for the health care and safety of children's sports activities.

\subsection{Lack of Physical Activity and Environmental Facilities Requirements}

The most direct influencing factors affecting the quality of children's sports activities are the sports facilities in the family, kindergarten and society environment, and the sports literacy of educators, facilities being strong determiners, and the sports literacy of educators being weaker determiners. Sports facilities, including kindergarten activity rooms, outdoor sports facilities and event venues are an important part of the entire educational environment. The key to improving the quality of children's sports activities is to improve the sports facilities in the environment and improve the sports literacy of educators. With continuous urban expansion across the country, the increase in the number of kindergartens has negatively affected the distribution of kindergarten resources. There are differences in the types, quantity and equipment of sports facilities between kindergartens of different grades and different schools, and also the expertise level of sports educators, and parents, in early years education is not high.

It is necessary to adopt corresponding measures to regulate the venues and equipment of kindergarten sports activities, and increase the investment in sports facilities for young children by formulating national standards, such as increasing the area of activity venues, optimizing the materials of the venues, and increasing the size of small and medium-sized sports equipment, especially encouraging selfmade sports equipment, making full use of community resources, and actively expanding the space for children's sports activities, etc., in order to improve the enthusiasm of children to participate in sports activities. Children's sports activities are related to the guidance of educators, which includes teachers and parents, and therefore improving teachers' sports literacy, encouraging parents to learn relevant educational theories, and strengthening communication between kindergartens and parents is of great significance to promote the healthy growth of children and the development of the body, and to reduce the occurrence of accidental injuries.

\subsection{Lack of Early Childhood Physical Education Professionals}

The physical education skill, curriculum organization ability, teaching ability and practical ability of kindergarten teachers are the core issues in the quality of children's physical education activities. The comprehensive quality of children's physical education professionals directly affects the effectiveness of children's sports activities. At present, preschool teachers have mostly graduated from junior colleges and are undergraduates. Most of them are graduates of preschool education. Although non-professional sports teachers understand the general characteristics and basic teaching methods of children's physical and mental development, they lack systematic learning and basic sports knowledge and skills. The name and the principle of motion technology are insufficiently understood. In our survey of kindergartens in Guangdong Province, we found that preschool teachers who graduated from physical education only accounted for $2 \%$ of kindergarten teachers. At present, many kindergartens use the method of external physical education teachers to make up for the shortage of physical education teachers, but they are still far from meeting the needs of children's sports activities. The quality of children's sports activities is difficult to guarantee. In some schools with better conditions, there will be professional physical education teachers, and private kindergartens are far from being able to adapt to the development needs of kindergarten sports activities due to insufficient funds. In the absence of kindergarten physical education professionals in kindergartens, the ability of preschool teachers to teach children's sports activities is greatly limited. Most kindergarten teachers will guide children's sports activities based on personal sports learning experience, lacking pertinence, professionalism and science.

\subsection{Main Reasons for the Unsatisfactory Development of Children's Sports Activities}

(i) The country does not really include pre-school education in the scope of compulsory education. The development of kindergartens and the pre-school education are far behind the other major countries in the world. The development of children's sports activities is only satisfied with the requirements, and there are no regulations on the content of sports activities, venues, equipment and teachers. The level of laissez-faire sports activities has reached a level of indulgence.

(ii) Although the "Development Guide" attaches importance to physical development and puts the development of young children's movements under the health field, and even requires children aged 3-6 to have a certain "balance of ability, coordination, sensitivity, strength and endurance, hand movement and flexible coordination", there is still a lack of syllabus or curriculum standards or general learning content for children's sports activities.

(iii) It has long been influenced by the education and physical education system of the former Soviet Union. Kindergarten teachers accept sports education system based on sports items. Therefore, the concept of "children's sports has always stayed in the concept of sports, and too many competitive sports items and practice methods have been used in kindergarten sports activities. Many kindergartens copy skills of sports programs; pure basketball, football 
technology teaching, and competitions abound." [16].

(iv) The rapid development of the society has affected the normal development of children's sports activities. In the environment of fierce competition in education, parents' awareness of "winning at the starting line" has helped the social kindergarten training institutions to organize various sports training courses.

\section{Conclusion and Reconfiguration}

At present, there is no unified physical education syllabus in kindergartens. The tendency of adult learning is to be serious, and the test evaluation criteria are broad. It has been plaguing the development of sports activities in kindergartens and even leads to sports safety issues. According to the requirements of the "Development Guide" and range of preschool children's physical activities, comprehensive research on the development of basic activity ability of children in all age groups (3-6 years old) is to be carried out, and the activities, exercise methods and evaluation methods for the basic activities of children in all age groups are to be established. Focused on promoting infants' balance, strength and endurance, agility and coordination, etc. We also need to construct a test system which can be use to establish an indicator system of the test and evaluation methods for preschool children. The development of the sports activity system will have a profound impact on the promotion of the normal development of children's sports activities and the healthy development of children's physical fitness.

\subsection{Stratification and Progressive Construction of Children's Sports Activity Content System}

According to the "Development Guide", the goals for young children are: a certain balance of ability; coordination of movement, sensitivity, certain strength and durability, and flexible coordination of hand movements, etc. Based on motor skills, it establishes a steerable sports system of different age stages, step by step, from easy to difficult displacement skills (such as running), non-displacement skills (such as twisting) and operational skills (such as throwing). The sports activity system consists of three aspects: 3-6 year old balance, coordination and sensitivity, strength and durability of the basic motor skills content system (such as walking: natural walking, forefoot walking, heel walking, small walking, high lifting of the legs, walking, springing, retreating, changing arm movements, slamming, holding things, walking together, obstacles, walking straight, walking parallel lines, wood balancing walking); 3-6 year old basic ball movement skill activity system (such as 3-4 years old, a variety of homemade ball equipment simple different gameplay, free to choose and pick and place materials; 4-5 year olds beginning to cooperate with peers in cooperative ball games; 5-6 year olds having independent subjective consciousness and consciously positive learning attitude and creating different gameplay in the game process.); and a kindergarten outdoor sports activity content system (such as outdoor sports activities mainly based on balance equipment, coordination sensitive equipment and power endurance equipment).

\subsection{Screening and Establishing a Measurement Index System for Children's Sports Activities}

The development of children's physical fitness can be expressed in life and in execution of different body movements. For the content of sports activities of children of different ages, it is necessary to screen and establish the measurement index system and evaluation method for children's sports activities of different ages. Quantitative criteria are given for the measurement of large muscles in children of all ages, and the development of physical fitness is clearly quantified by the amount and duration of movement performed by the child.

At present, the measurement and evaluation system for children's physical activity widely used abroad is "Test of Gross Motor Development 2" (TGMD 2). TGMD 2's large muscle movement development test includes both the "displacement action test" and "object control action test", which are specifically used to evaluate the development of large muscle movements in children aged 3-10 years. Displacement motion measures the flexibility, coordination, and graceful movement of large muscle groups required for the child to move from one direction to the other. This includes running, front sliding, one foot jumping, cross jump, standing long jump, and the side sliding step. The object control action test measures the ability of large muscle groups to exercise effectively by throwing, picking, and striking. This includes hitting the ball, keeping the ball in place, catching the ball with both hands, kicking the ball, pitching the ball, and rolling the ball. It has been designed to assess the developmental role of large muscle groups in children aged 3-10 years and has been proven to be highly reliable and effective [17].

On the basis of constructing scientific content and methods of children's sports activities, through tracking, testing, experiment and data statistic analysis, the age groups and various activities can be expanded to form the measurement and evaluation criteria for greater improvements in children's sports activities. Finally, a relatively complete evaluation system for measuring indicators of children's sports activities is formed.

\subsection{Creating a Suitable Sports Game Environment}

Exercise can occur in any natural environment. The welldesigned sports game environment is provides children with sufficient indoor and outdoor sports space, fully meets the needs of children to learn motor skills, and promotes the development of sports ability of all children. As a material condition for children's sports activities, sports facilities directly affect the physical development and exercise effects for young children. Kindergartens which cater for children's sports activities, games and play, and the production of quality equipment should be a priority, and the size and weight of sports equipment in the environment should be 
changed according to children's sports needs, physical fitness and activities. The establishment by families, local community, teachers, parents and other adults to create a safe sports environment for children's sports activities and exercise health care can extend to monitoring the amount of children's exercise and encouraging children to succeed in the field of sports.

\subsection{Establishing a Teaching Organization System for Children's Sports Activities}

Because young children do not have complete selfdevelopmental ability, the maintenance and promotion of children's physical health depends on the education level of kindergarten teachers and parents. The professional quality and practical ability of kindergarten teachers are important human guarantees for the quality of early childhood education. Teaching activities are organized by educators, including teachers, parents, and children. Different physical activity programs are taught by different teachers to produce different teaching effects for young children. Enhancing educators' sports literacy and guiding skills can enable young children to receive both scientific and effective physical activity guidance, promote healthy growth of young children, and enable children to enter into participation. The habit formation of physical exercise can therefore find an earlier starting point in school physical education.

\subsection{Pay Attention to Children with Special Needs in Sports Activities}

In recent years, "integrated education" and "inclusive education" have been carried out in the field of special education at home and abroad. The entry of special children into ordinary kindergartens and enjoying the same education as normal children has become a basic trend of preschool education development. Under the background of promoting integrated education in our country, based on the professional development of kindergarten teachers, we will improve the awareness of kindergarten teachers on physical education of children with different characteristics, so that they have the basic ability to develop personalized education programs for young children with special needs. Children's right to exercise is needed to improve the overall quality of children with special needs, to promote the healthy and comprehensive development of all young children, to enable children to develop the habit of participating in physical exercise and to have a higher starting point in school physical education.

\subsection{Cultivation of Children's Behavioral Norms and Rules Awareness}

Codes of conduct and social rules are the norms that people must abide by in social life learning and work, and play a very important role in social life. Studies have shown that 3-5-year-old children's cognition of social rules comes from their actual social interactions, and presents a dynamic process of continuous development. That is to say, with the increase of age, young children can learn to distinguish more social rule events, and the understanding of the concept of rules is further deepened. The compliance and enforcement of rules gradually shift from the other to more self-discipline, thus internalizing into their own actions [18]. The ability to develop children's rules and the ability to enforce them from an early age helps young children adapt to social life in their adulthood. By formulating sports game rules, children can be raised and cultivated in their activities to develop and understand behavioral norms and rules.

\subsection{Integration of Sensory Training in Early Childhood Sports Activities}

The theory of sensory integration was first systematically proposed in 1969 by Ayres A. J, Ph. D. in clinical psychology at the University of Southern California. So-called sensory integration means that the human brain will analyze and comprehensively process the sensory information transmitted from various sensory organs, and make an appropriate response, so that the individual can operate harmoniously and effectively in the stimulation of the external environment. The average child establishes this dominant brain before the age of 5. If the dominant brain fails to establish, there will be a "sensory disorder", resulting in poor balance between the body and the muscles in the future, which is characterized by clumsiness, poor flexibility, and hyperactivity and leads to inattention, distraction and personality problems (impulsiveness, willfulness, temper). Sensory integration disorders are threatening the mental health of children in our country, especially in economically developed areas. According to incomplete statistics, $10 \%-30 \%$ of children in China currently suffer from this disease to varying degrees. A survey of 2031 school-age children in Shanghai showed that the rate of mild sensory integration disorders in children was $36.6 \%$, and the severity was $16.1 \%$. Recently, the affiliated hospital of Nanjing Railway Medical College surveyed 2486 people aged 6 to 11 in Nanjing. Through school-based and systematic tests, school-age children found that $34.9 \%$ of school-age children had different levels of sensory integration disorders. The experimental research shows that the sensory integration training course combined with the children's physical activity curriculum has a positive effect on the physical quality of children, and it provides very obvious help for children's all-round growth [19].

\section{Outlook}

Early childhood education is an important part of China's socialist education, the foundation of China's basic education, and the Mochi project to improve the quality of the whole people. It plays a decisive role in cultivating the awareness, skills and habits of young children's physical exercise. Based on the principles of applicability, science, interest, safety, etc., we will construct a sports activity system that conforms to the laws governing the development of children's mind and body. We will act according to the law of children's physical and mental development, and design activities and evaluation 
systems based on the basic development level of children's movements to prevent adult children. Chemical education provides reference and reference for promoting the reform and development of children's physical education, which is conducive to improving the overall level of children's physical education in China and promoting the physical health of young children.

\section{References}

[1] Ministry of Education. Guidelines for Kindergarten Education (Trial) [Z], 2001.3.

[2] Greg Payne, Pei Peixin, Liang Guoli. Introduction to Human Movement Development [M]. Beijing: People's Education Press. 2008.

[3] Ministry of Education. Kindergarten Work Regulations [Z], 1996: 1 .

[4] Ministry of Education. National Medium- and Long-Term Education Reform and Development Plan (2010-2020) [Z], 2010.

[5] Aristotle (Ancient Greece). Political Science. Volume 7. Kyushu Press. 2007.

[6] Huo Liyan's Comparative Study of Preschool Education System in America, Britain, Japan and India [M].

[7] Zhou Cai. Compare preschool education. [M]. Beijing. People's Education Press. 2013.

[8] $\mathrm{Xu}$ Zhuoya. Preschool Children's Sports. [M]. Beijing. Nanjing Normal University Press. 2003.10
[9] Liu Xin. Preschool children's sports. [M]. Beijing. Beijing Normal University Press 2014.2

[10] Pang Jianping, Liu Qian. Preschool Children's Health Education and Activity Guidance. [M]. Shanghai. East China Normal University Press, 2014.3

[11] Wang Zhanchun, Chen Yiqi, "Theories and Methods of Kindergarten Sports Activities". [M]. Beijing. People's Education Press, 2011.

[12] Ministry of Education. Guidelines for Learning and Development of Children 3-6 Years [Z], 2012.

[13] Zhang Ying. Empirical Research on the Contents of Children's Sports Activities from the Perspective of Action Development [J]. Journal of Beijing Sport University, 2012, 35 (3): 133-140.

[14] Geng Peixin, Liang Guoli. Introduction to Human Movement Development [M]. Beijing. People's Education Press. 2008.

[15] State Sports General Administration. "National Physical Fitness Measurement Standards (Children's Part)" [M]. People's Sports Publishing House, Beijing. 2003.

[16] Zhuang Bi. Taking the body as the first place to lay the foundation for the healthy development of children. [J]. China School Sports. 2014.11.

[17] Li Jing, Liang Guoli. Large muscle group development test TGMD2 research [J]. China Sports Science and Technology, 2005, 41 (2): 105-107.

[18] Yue Xuntao. Cognitive Characteristics of Children's Social Rules and Cultivation of Game Rules Consciousness [J]. Basic Education Research, 2012, 11: 58-59.

[19] You Fuyu. The status quo of sensory integration training courses and its impact on children's physical fitness [D]. Sichuan Normal University, 2011. 